\title{
EFFECTIVE PAIR INTERACTION FROM $A B$ INITIO PSEUDOPOTENTIALS - IMPORTANCE AND APPLICATIONS
}

\author{
S. HALDAR \\ Condensed Matter Physics Research Centre, Physics Dept. \\ Jadavpur University, Calcutta - 700 032, India \\ A. SARKAR \\ B.K. Girls' College, Howrah 711101, India \\ AND D. SEN \\ Barasat Govt. College, Barasat 743201, West Bengal, India \\ (Received August 12, 1999; in final form January 12, 2000)
}

\begin{abstract}
Computation of reliable effective interionic pair interaction for simple metals has been discussed on the basis of localised $a b$ initio pseudopotential within linear screening approximation. The influence of different commonly used screening functions on the shape of pair potential is examined and the temperature dependence of the interaction is also investigated. Various features of the potential are studied and compared with empirical and available first principle results. Importance of the present study and some related problems are also analysed.
\end{abstract}

PACS numbers: $63.10 .+\mathrm{a}, 65.90 .+\mathrm{i}$

\section{Introduction}

Since the seminal contribution of March and coworkers [1], considerable progress has been made in the development of the effective interionic pair potential approach in condensed matter research. A real space description of bonding energetics in terms of effective interaction by "integrating out" a number of microscopic degrees of freedom is useful in material physics for several reasons. Besides providing improved interpretability and direct understanding of structural stability and relaxation phenomena in simpler terms, it offers viable computational schemes for simulation of complex material science problems. Ever expanding computing capabilities notwithstanding, the desired computational speed essential for com- 
puter simulation requires schemes of comparable simplicity. Useful applications of interionic potential methods are being made in defect properties, thermodynamic properties of solids and liquids, structure and dynamics of expanded liquid systems, surface studies and molecular-dynamics (MD) to name important few. More sophisticated and amvitious task of including simultaneously the ionic and electronic degrees of freedom in the $a b$ initio molecular dynamics study [2] requires enormous computational effort. The size of the system which can be afforded in this scheme is generally much smaller compared to that in classical simulation. Although, the introduction of ultrasoft pseudopotentials have somewhat extended the scope of the scheme [3], the technique is extremely expensive in terms of CPU time to get wider applications to first-row elements and transition metals. Furthermore, the pair potential based MD calculations also provide useful standard for comparison with the first principle MD studies and in a recent first principle approach [4] final result of the former is used as the initial choice of the ionic configuration.

In the limit of adiabatic approximation, the Born-Oppenheimer (BO) theory predicts that the internal energy of a solid (condensed phase) can be broken up into a sum of two-body, three-body etc. energies. The successive terms are expected to be of decreasing importance. The pseudopotential theory of metals, however, suggests the possibility of a break-up of the total metallic energy into a pure volume dependent part plus an energy due to density dependent effective interionic interactions. The volume dependent energy in the pseudopotential theory may be viewed as a smeared out many-body interaction. The total energy of a metal, according to second-order pseudopotential theory, may be expressed in terms of a density dependent pairwise interionic interaction in addition to the volume dependent energy. Higher order energy terms actually contain, in addition to some residual volume energy and two-body, interactions among three and more ions.

Pair potentials cannot by themselves describe the broad range of material properties of transition metals and semiconductors [5]. However, most simple metals and simple metal based binary systems appear to be well described at the pair potential level and these potentials are being used in atomistic simulations. Molecular dynamics result for liquid Ge shows that the structure of this metallic liquid is well described in terms of volume forces and pair potentials without invoking explicit many body forces [6]. A large number of atomistic simulations have also been performed for transition metals using only pair potentials with parameters fitted to several known properties [5].

Several calculations are made where pure two-body potentials are obtained from direct inversion of the total cohesive energy by the forcible subsumming of the many-body effects $[7,8]$, while some attempts have also been made to include explicit three- and many-body potentials in the cohesive energy expression $[9,10]$. However, these are in conflict with the pseudopotential theory of metals and introduce artificiality in the description. For example, the parametrized fitting scheme of Kluge et al. [10] gives a counterintuitive result that the three-body energy contribution is larger in the liquid and amorphous phase than that in the crystalline phase. In order to obtain interionic potentials valid over a wide range of density, 
appropriate density dependence of the cohesive energy should have to be included. The pseudopotential approach, apart from being a microscopic theory, includes important many-body effects even in the second-order approximation through both purely volume dependent energy term and the parametric volume dependence of the effective pair interaction. Moreover, optimum transferability among a variety of geometries and chemical environments of the $a b$ initio pseudopotentials (AP) $[11,12]$ makes them particularly useful in deriving interionic potentials suitable for studies of defect properties. Combined with the present day accuracy of $a b$ initio pseudopotentials [13], this definitely underscores the importance of the search of a reliable interionic potential on the basis of them.

Studies with model pseudopotentials predict oscillatory two-body interaction with deep negative (attractive) minimum favouring a hard-sphere description for most simple metals. Understandably though, there are large variations [14-16] among various calculations with model pseudopotentials derived from piecemeal studies of different sets of few lattice mechanical properties. Interionic pair potentials obtained from comprehensive unified study can only claim reliability and indeed they are found to compare well [14] with the few first-principle calculations made so far. However, the positions and the values of the potential minima obtained from the model pseudopotential calculations compare rather less favourably with $a b$ initio calculations [17-19]. Also, in alloys model pseudopotentials usually fail [6] and one has to use more reliable $a b$ initio psedopotentials. The calculation of electron-ion correlation function $g_{\mathrm{ei}}(r)$ (within the linear screening approximation assumed for the valence electron density around each ion) provides another test for the pseudopotential itself. The position of the first minimum of $g_{\mathrm{ei}}(r)$ is almost the same as that of the first peak of ion-ion pair correlation function $g(r)$ [4]. Calculation with ab initio pseudopotential [11,20] for Al shows (Fig. 1) much better agreement with the empirical data [21] compared even to that with model (pseudo) potential of earlier unified study [14].

$A b$ initio approaches provide a physical insight of the problem as well as a microscopic theoretic understanding of the various approximations made. In the present work we intend to calculate the effective interionic pair potentials of the

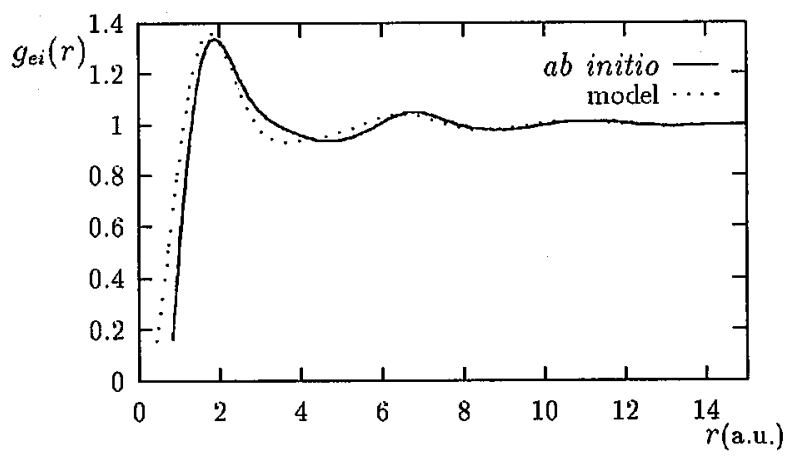

Fig. 1. The electron-ion pair correlation function $g_{\mathrm{ei}}(r)$ for $\mathrm{Al}$. 
alkali metals and those of polyvalent $\mathrm{Mg}$ and $\mathrm{Al}$ with the localised ab initio pseudopotential form factors derived in Ref. [20] as the first part of our programme for the determination of reliable pair interaction for a host of other group of metals. The dependence on various screening functions and variation with temperature have also been investigated systematically. Changes in the pair potential on alloying occur from the variation of the electron density dependent screening function and due to variation of pseudopotential with composition. The pair potential derived from pseudopotential theory is a "constant volume" interionic potential suitable for homogeneous systems and describes ionic rearrangements at constant volume. However, Matsuda et al. [22] have studied the dependence of the structure of expanded liquid alkali metals over a wide range of densities based on the effective pair potentials derived from electron-ion pseudopotential within the linear screening approximation and found excellent agreement with experimental results. Pair potential in strongly inhomogeneous system, may be described in terms of a local density [23] derived from an average over some finite distance comparable to the electronic screening length. In the solution of the compressibility problem the concept of local volume was introduced earlier [24, 25] with a specific form for the local volume strain, satisfying some general properties demanded by the lattice mechanical theory. The force constant elements redefined with some additional terms, ensure the equivalence of the compressibility and other elastic constants in "homogeneous deformation" and "long wave" methods [24]. Using the concept of local volume it may be, therefore, possible to describe ionic rearrangements under varying local volume keeping the average volume constant.

The study of temperature dependence of the dynamical structure of expanded liquid is generally based on the effective pair potential. Different temperature conditions influence the interaction through the change in the atomic volume. Empirical studies have shown a "normal ordering" that the first minimum of $\phi(r)$ becomes deeper with increasing temperature without much affecting the position of the minimum [26]. A systematic study of variation of the pair potential curve with temperature is, therefore, very important. Precise form of the screening function has a drastic influence on the shape of the effective pair potential at low and moderate distances and is found to affect the "normal temperature ordering". In a previous work Sarkar and Sen [27] have briefly reported applications of interionic pair potential of rubidium using localised $a b$ initio form factor [20] with reasonable success. The results show the importance of using first-principle pseudopotential in determining reliable effective pair potential for metals.

\section{Basic theory}

Simple metal effective interionic pair potential according to second-order pseudopotential theory is given by

$$
\phi(r)=\frac{(Z e)^{2}}{r}-\frac{\Omega}{\pi^{2}} \int_{0}^{\infty} F_{N}(q ; \Omega) \frac{\sin (q r)}{q r} q^{2} \mathrm{~d} q,
$$

where $Z$ is the valence and $F_{N}$ is the Harrison [28] energy-wave number characteristic function that embodies detailed electronic band-structure effect including the self-consistent electron screening. Pseudopotential form factors involved in $F_{N}$ are 
plane wave matrix elements of ion-electron pseudopotential. The nonlocal ab initio pseudopotential is formally written as

$$
\widehat{V}=V(r)+\sum_{l} V_{l}(r) \hat{P}_{l},
$$

where $\hat{P}_{l}$ is the projection operator on angular momentum $l$. The analytical forms for $V(r)$ and $V_{l}$ are given in Ref. [11]: The generalized form factor consists of the Fourier transform of the local part $V(r)$ and the matrix element (between the initial $\left|k_{\mathrm{i}}\right\rangle$ and the final $\left|k_{\mathrm{f}}\right\rangle$ plane-wave states) of the purely nonlocal part $V^{\mathrm{NL}}$, which is given by

$$
\left\langle k_{\mathrm{f}}\left|\widehat{V}^{\mathrm{NL}}\right| k_{\mathrm{f}}\right\rangle=\frac{4 \pi}{\Omega} \sum_{l}(2 l+1) P_{l}(\cos \theta) \int V_{l}(r) j_{l}\left(\left|k_{\mathrm{i}}\right| r\right) j_{l}\left(\left|k_{\mathrm{f}}\right| r\right) r^{2} \mathrm{~d} r
$$

where $j_{l}$ and $P_{l}$ are spherical Bessel functions and Legendre polynomials respectively, with

$$
\theta=\angle k_{\mathrm{i}}, k_{\mathrm{f}} \text {. }
$$

The usual local approximation retains only the "on Fermi sphere" (OFS) backscattering terms in Eq. (3). Total emphasis on backscattering gives form factors that result in a large value of resistivity (almost double) for simple metals such as $\mathrm{Na}, \mathrm{Mg}$, and $\mathrm{Al}[20]$. A new local approximation (beyond OFS) makes an angular a verage of the matrix element of the nonlocal potential (Eq. (3)) considering scattering in all possible directions (on and off the Fermi sphere). The average localised form factor $v_{q}$ so obtained is considerably short ranged (more than $25 \%$ reduction of the form factor at the first maximum near $1.9 k_{\mathrm{f}}$ for $\mathrm{Al}$ compared to the OFS value), smooth and appears to retain some essential nonlocal features [27]. With linear dielectric screening that takes into account the exchange-correlation $(\mathrm{XC})$ functional $G(q)$ [29], perturbative calculations using these form factors are found to give an excellent account of wide range of properties of simple metals [20].

The precise form of the XC functional $G(q)$ in the dielectric screening function has a pronounced effect on the pair potential curve. Various analytic expressions for $G(q)$ have been proposed satisfying a number of self-consistency criteria. In the present study we have used four different forms to investigate the influence of screening function on $\phi(r)$. It is generally appreciated that the modified Ichimaru and Utsumi (IU) [30] form gives a good account of XC functional for degenerate electron gas at metallic and lower densities. However, certain inadequacies are subsequently discussed in current literature and in a recent formulation [31] a simple parametric form, free from various inconsistencies has been proposed. A very accurate fit of electron correlation energy data in the entire density range is used for the calculation of $G(q)$ which produces some interesting results. The present study also provides us with the opportunity of judging the merit of different XC functionals. The variation of $\phi(r)$ with temperature is also studied using temperature variation of the atomic volume $(\Omega)$.

In the high temperature limit $\left(T \gg T_{\mathrm{m}}\right)$, the ionic pair correlation function may be approximated [32] as

$$
g(r)=\mathrm{e}^{-\phi(r) / k T} .
$$


The ancillary hard-sphere description [33] of interionic pair interaction defines the relevant parameter i.e. the hard-sphere radius $(\sigma)$ as

$$
\phi(\sigma)-\phi_{\min }=\frac{3}{2} k T .
$$

Empirical value of the packing fraction $\eta\left(=\pi \rho \sigma^{3} / 6\right)$ determined from structural data [21] may be compared with the theoretical value using $\sigma$ determined from Eq. (5).

The contribution of the third-order perturbation energy term to the pair interaction is discussed earlier by Hasegawa [34]. The formulation has been completed with the inclusion of a missing term [35] and will be discussed elsewhere.

\section{Results and discussion}

The problem of inferring pair interactions to good accuracy starting from structural data can be regarded as solved in the case of classical liquids [36]. However, the method requires high accuracy of structure factor data over extended range of $q$-values. We, therefore, refrain from any thorough comparison of the present calculation with the "empirical" potential obtained from the structural data.

The effective pair potentials computed with localised $a b$ initio pseudopotential in second-order theory for simple metals like $\mathrm{Li}, \mathrm{Na}, \mathrm{K}, \mathrm{Rb}, \mathrm{Mg}$, and $\mathrm{Al}$ are presented first. Calculations are made with four different screening functions of Geldart-Vosko (GV) [29], Taylor (T) [37], IU [30] and the recently proposed one by Sarkar, Sen, Haldar and Roy (SSHR) [31]. The calculated phonon spectra from the computed pair potentials are found to agree with earlier second-order pseudopotential calculation [20].

The nature of variations of the pair potentials for six metals with four different screening functions are obtained. Very similar values are found with IU and $T$ screening functions where as SSHR and GV forms produce closer results. The variations are more pronounced for polyvalent metals as expected (Fig. 2a, b). From the calculated temperature variations of $\phi(r)$ we note that the screening functions of $\mathrm{T}$ and IU do not produce the "normal" temperature ordering in case of $\mathrm{Li}, \mathrm{K}$, and $\mathrm{Rb}$. The density values at different temperatures are taken from Refs. [21] and [22]. Temperature variations are comparatively large in cases of $\mathrm{Na}, \mathrm{Mg}$, and Al [38].

For all four alkali metals and $\mathrm{Mg}$, our second-order calculations of $\phi(r)$ exhibit just beyond the core deep attractive minima close to the nearest neighbour distances and at large distances usual Friedel or Ruderman-Kittel oscillations with single dominant wavelength $\lambda\left(=\pi / k_{\mathrm{f}}\right)$ characteristic of sharp Fermi-surfaces in metals. Except for $\mathrm{Li}$, the positions of the first minima of the potentials agree with the other two first-principle calculations (Ref. [17] and [18]). However, there are discrepancies regarding the positions of the first zeros and the depth of the minima of the potentials (Fig. 3a-c). The pair potentials obtained by Rasolt et al. from their self-consistent nonlinear charge density calculations show that, of all the alkali metals, Li has the strongest attractive pair potential and lack of any sort of ordering from $\mathrm{Li}$ to $\mathrm{Rb}$. This is somewhat surprising and contrary to what our results show - a systematic increase in potential depth from $\mathrm{Li}$ to $\mathrm{Rb}$. 

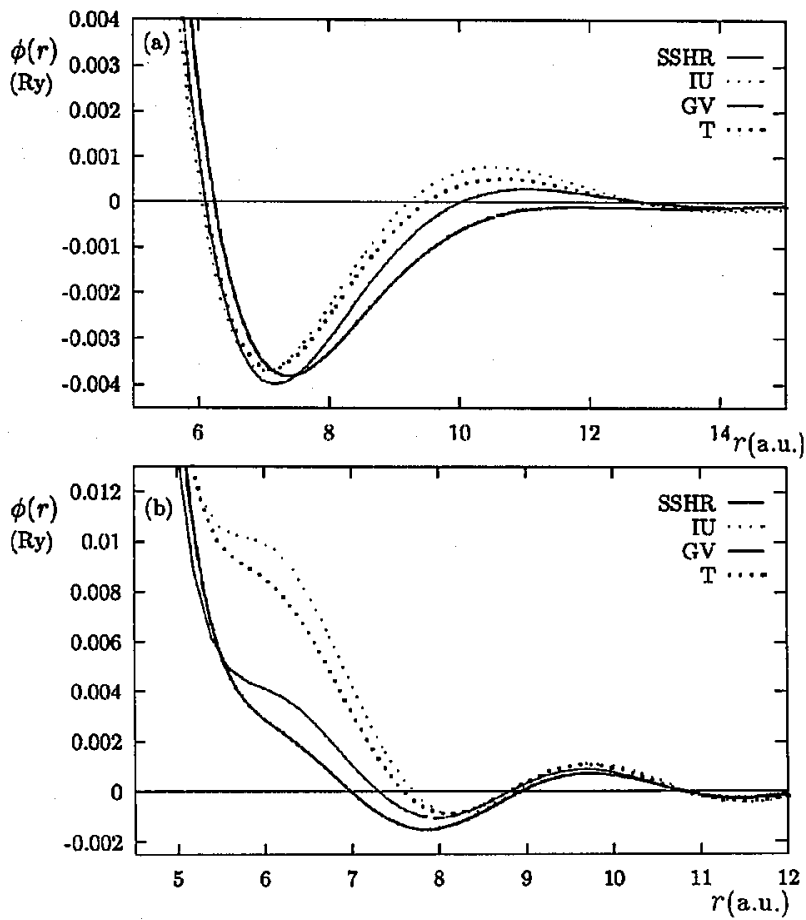

Fig. 2. The nature of variations of the pair potentials $\phi(r)$ with four different screening functions for (a) $\mathrm{Na}$ and (b) Al.*

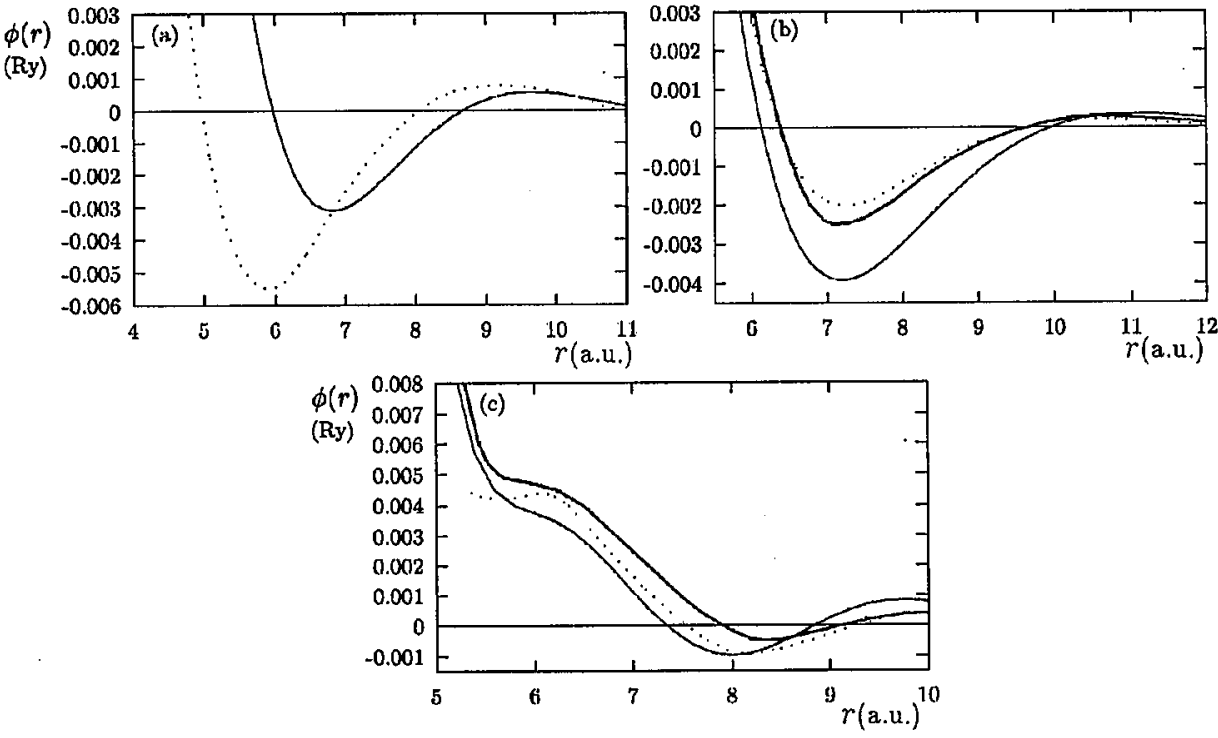

Fig. 3. Comparison of the pair potentials with other ab initio calculations. (a) $\mathrm{Li}$, (b) Na, and (c) Al. Thin solid curves represent present calculation, dotted curves are due to Dagens et al. [17] and bold solid curves - from Ref. [18]. 
The pair correlation function determined with second-order $\phi(r)$ at high temperature limit $\left(T \gg T_{\mathrm{m}}\right)$ for all the metals excepting Al are shown to compare well (Fig. 4) with the existing Monte Carlo calculations [22]. It may be noted that the static structure factors obtained from the effective pair potential for $\mathrm{Rb}$ near melting temperature by an iterative scheme [27] are in excellent agreement with the calculated and the experimental results [39]. The theoretical values of hard-sphere radii are also found to agree well with the empirically determined values and are shown in Table. Here also we note better agreement obtained with SSHR and GV screening functions. For Li, hard-sphere radius given by Rasolt et al. potential appears to be widely different from the empirical value. Much importance should not be attached to this rather surprisingly good agreement with

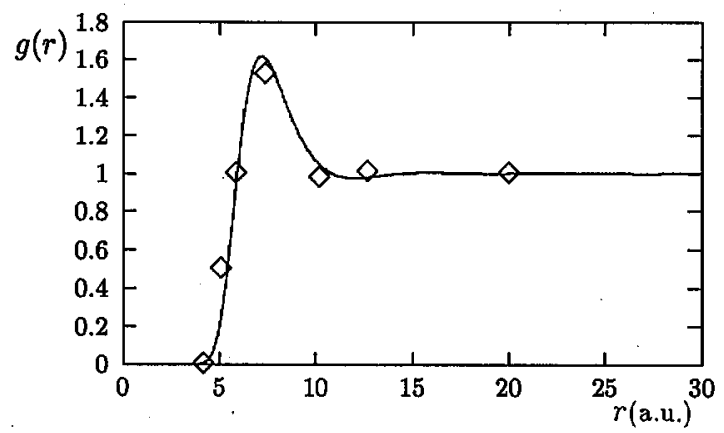

Fig. 4. The ion-ion pair correlation function $g(r)$ at high temperature limit for $\mathrm{Na}$ $(2000 \mathrm{~K})$. Solid curves represent present calculation and diamonds are from Ref. [22].

\section{TABLE}

Temperature variations of calculated and empirically determined values of $\sigma$.

\begin{tabular}{c|c|c|c}
\hline \hline Element & Temp. [K] & $\sigma$ (cal.) [a.u.] & $\sigma$ (empirical) [a.u.] \\
\hline $\mathrm{Li}$ & 520 & 5.1 & 5.1 \\
& 1000 & 5 & - \\
$\mathrm{Na}$ & 380 & 6.2 & 6.3 \\
& 820 & 5.9 & 6 \\
$\mathrm{~K}$ & 340 & 8 & 7.6 \\
& 720 & 7.5 & 7.34 \\
$\mathrm{Rb}$ & 350 & 8.4 & 8.1 \\
& 900 & 8.2 & 7.84 \\
$\mathrm{Mg}$ & 1153 & 5.5 & 5.48
\end{tabular}


these second-order calculations. Firstly, the third-order term contributes substantially to the calculation of $\phi(r)$ and worsens the overall agreement to some extent. Secondly, for the present $a b$ initio pseudopotential, 3-body interaction implied in the third-order term is also not negligible. However, it is clear that the potentials of Refs. [17] and [18] fail to produce reasonable agreement in the case of pair correlation function.

It is regarded that some higher order contributions get included $[5,34]$ in the pair interaction scheme such as one by Rasolt et al. But then, their result for $\mathrm{Al}$ is very similar to our second-order calculation (Fig. 3c). For Al, there is something of a ledge structure in the repulsive region around the nearest neighbour distance and only vestiges of an attractive minimum at the second neighbour distance indicating deficiency of a hard-sphere description. On the other hand, adopting closely similar procedure Manninen et al. [19] have obtained $\phi(r)$ for $\mathrm{Al}$ (inserted in a jellium vacancy) which is better described by a hard-sphere model. Very recently Mishin et al. [40] have developed interatomic potential for $\mathrm{Al}$, using embedded atom method (EAM) potential functional based on a large set of experimental and $a b$ initio data, which gives pronounced hard-sphere description. Simple argument
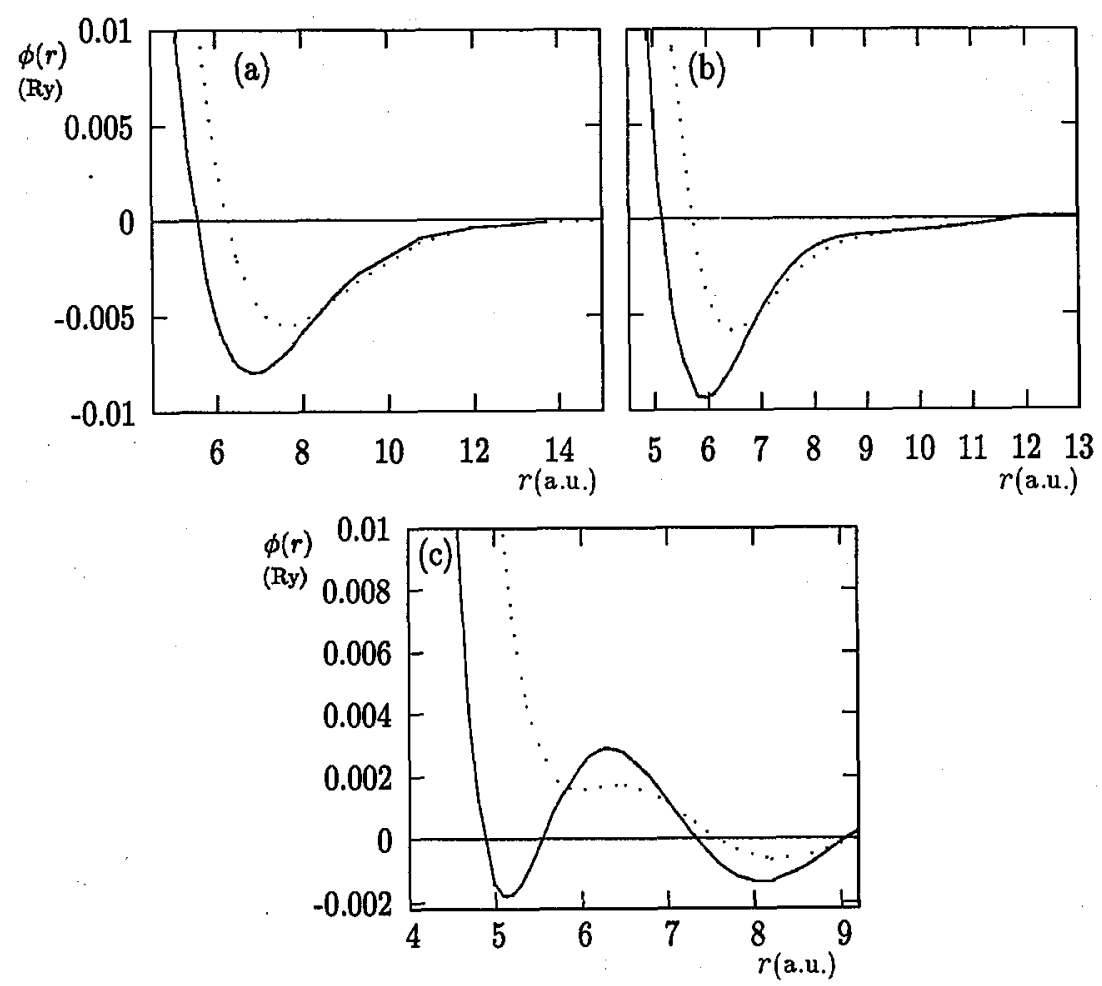

Fig. 5. Comparison of pair potentials obtained from calculations up to 2nd- and 3rd-order for: (a) $\mathrm{Na}(2000 \mathrm{~K}),(b) \mathrm{Mg}(1153 \mathrm{~K})$ and (c) Al (1323 K). Thin solid curves represent 3rd-order calculations and dotted curves represent 2nd-order calculations. 


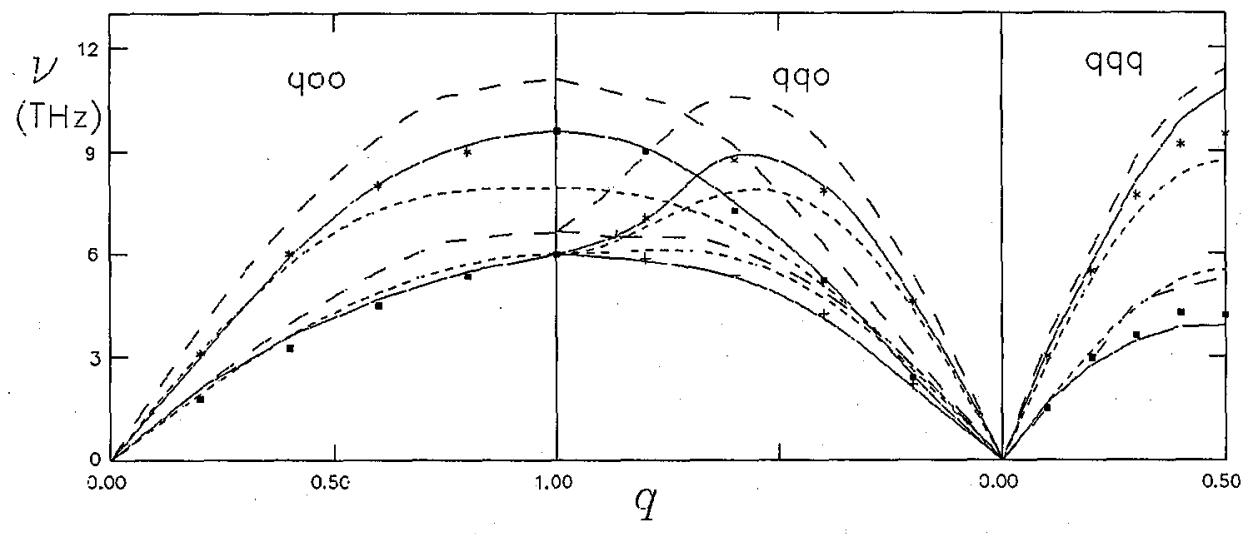

Fig. 6. Phonon dispersion curves obtained from pair potentials calculated up to 2ndand 3rd-order and from full 3rd-order pseudopotential calculations for Al. Solid curves represent pseudopotential calculation, small dashed and long dashed curves represent calculations from 3rd- and 2nd-order pair potentials respectively. Experimental points are indicated by stars, solid squares and plus symbols for longitudinal and two transverse branches respectively.

based on the validity of Eq. (4) for the high temperature $\left(T \gg T_{\mathrm{m}}\right)$ pair correlation function of $\mathrm{Al}$ also favours similar description for $\phi(r)$.

Additional pair potential due to third-order perturbation term in electron-ion interaction is comparatively large and attractive at short distances and shifts the first zero of the potential to smaller distances by several percent $[34,35]$.

For alkali metals and $\mathrm{Mg}$, however, no qualitative change occurs in the effective pair interaction with the inclusion of third-order contribution (Fig. 5a, b). But for $\mathrm{Al}$, the third-order contribution drastically alters the pair potential and for $T \gg T_{\mathrm{m}}$, a hard-sphere description in conformity with the empirical ionic pair correlation data emerges (Fig. 5c). Figure 6 depicts the phonon dispersion curves obtained from the pair potential and compared with the full third-order pseudopotential $a b$ initio) calculation. The comparison provides an estimate of the 3 -body contribution to lattice vibration in simple metals.

Full third-order calculations which include up to explicit 3-body interactions only show reasonable agreement with the experimental results for Al. Our calculation up to second order overestimates phonon frequencies in all the symmetry directions whereas inclusion of the third-order 2-body (small dashed curves in Fig. 6) lowers the frequencies much below the experimental values. Contributions of the 3-body interaction turn out to be significant except for [q00] and lower [qq0] transverse branches. Near the zone boundary, the 3-body contributions to the [q00] longitudinal and the higher [qq0] transverse branches are as high as 15 to $20 \%$. For the $[q q 0]$ longitudinal branch the 3 -body contribution of about $15 \%$ is obtained near $(0.6,0.6,0)$ point.

\section{Acknowledgment}

One of the authors (S.H.) acknowledges financial support from the University Grant Commission, India. 


\section{References}

[1] M.D. Johnson, N.H. March, Phys. Lett. 3, 313 (1963); M.D. Johnson, P. Hutchison, N.H. March, Proc. R. Soc. A 282, 283 (1964).

[2] R. Car, M. Parrinello, Phys. Rev. Lett. 55, 2471 (1985).

[3] K. Laasonen, A. Pasquarello, R. Car, C. Lee, D. Vanderbilt, Phys. Rev. B 47, 10142 (1993).

[4] F. Shimojo, Y. Zempo, K. Hoshino, M. Watabe, Phys. Rev. B 52, 9320 (1995).

[5] A.E. Carlsson, Solid State Physics, Eds. H. Ehrenreich, D. Turnbull, Vol. 43, Academic Press, New York 1990, p. 90.

[6] J. Hafner, S.S. Jaswal, Philos. Mag. A 58, 61 (1988).

[7] A.E. Carlsson, C.D. Gelatt, H. Ehrenreich, Philos. Mag. A 41, 241 (1980).

[8] A. Mookerjee, N.-X. Chen, V. Kumar, Md.A. Sattar, Technical Report (IC/91/351), ICTP, Trieste 1991.

[9] F.H. Stillinger, T.W. Weber, Phys. Rev. B 31, 5262 (1985).

[10] M.D. Kluge, J.R. Ray, A. Rahman, Phys. Rev. B 36, 4234 (1987).

[11] G.B. Bachlet, D.R. Hamann, M. Schluter, Phys. Rev. B 26, 4199 (1982).

[12] D. Vanderbilt, Phys. Rev. B 41, 7892 (1990).

[13] R.M. Wentzcovitch, M.L. Cohen, Phys. Rev. B 37, 5571 (1988).

[14] D. Sen, Ph.D. thesis, University of Calcutta, 1982, unpublished; A. Sarkar, D. Sen, S. Sengupta, Pramana 20, 491 (1983).

[15] D. Sen, A. Sarkar, S. Sengupta, Acta Phys. Pol. A 67, 773 (1985).

[16] B.J.C. Cabral, J.L. Martins, Phys. Rev. B 51, 872 (1995).

[17] M. Rasolt, R. Taylor, Phys. Rev. B 11, 2717 (1975); L. Dagens, M. Rasolt, R. Taylor, ibid., p. 2726.

[18] A.K. McMahan, J.A. Moriarty, Phys. Rev. B 27, 3235 (1983); J.A. Moriarty, M. Widom, Phys. Rev. B 56, 7905 (1997).

[19] M. Manninen, P. Jena, R.M. Nieminen, J.K. Lee, Phys. Rev. B 24, 7057 (1981).

[20] D. Sen, Phys. Rev. B 42, 1217 (1990); D. Sen, in: Proc. Nucl. Solid State Phys. Symp., Bhopal 1988, Vol. 31C, DAE, Bhopal 1988, p. 115.

[21] Y. Waseda, The structure of Non-crystalline Materials, McGraw-Hill, New York 1980.

[22] N. Matsuda, H. Mori, K. Hosino, M. Watabe, J. Phys., Condens. Matter 3, 827 (1991).

[23] R.J. Harrison, Surf. Sci. 144, 215 (1984).

[24] D. Sen, S.K. Sarkar, D. Roy, S. Sengupta, Phys. Rev. B 24, 876 (1981); D. Sen, S.K. Sarkar, S. Sengupta, D. Roy, Phys. Status Solidi 115, 593 (1983).

[25] S.K. Sarkar, S.K. Das, D. Roy, S. Sengupta, Phys. Status Solidi B 83, 615 (1977).

[26] J. Hafner, J. Phys. F, Metal Phys. 5, 1439 (1975).

[27] A. Sarkar, D. Sen, Acta Phys. Pol. A 91, 1081 (1997).

[28] W.A. Harrison, Pseudopotentials in the Theory of Metals, Benjamin Inc., New York 1966, p. 39.

[29] D.J.W. Geldart, S.H. Vosko, Can. J. Phys. 44, 2137 (1966).

[30] S. Ichimaru, K. Utsumi, Phys. Rev. B 24, 7385 (1981). 
[31] A. Sarkar, D. Sen, S. Haldar, D. Roy, Int. J. Mod. Phys. Lett. 12, 639 (1998).

[32] N.E. Cusack, The Physics of Structural Disordered Matter: an Introduction, IOP Publ. Ltd., Bristol 1987, p. 104.

[33] J. Hafner, Liquid Metals, Inst. Phys. Conf. Ser. No. 30, Bristol 1976, p. 102.

[34] M. Hasegawa, J. Phys. F, Metal Phys. 6, 649 (1976).

[35] D. Sen, A. Sarkar, S. Haldar, Condensed Matter Days, Jadavpur Univ,, Calcutta 1999.

[36] L. Reatto, Philos. Mag. 58, 37 (1988).

[37] R. Taylor, J. Phys. F 8, 1699 (1978).

[38] S. Haldar, unpublished.

[39] K. Hoshino, H. Ugawa, M. Watabe, J. Phys. Soc. Japan 61, 2182 (1992).

[40] Y. Mishin, D. Farkas, M.J. Mehl, D.A. Papaconstantopoulos, Phys. Rev. B 59, 3393 (1999). 